QUARTERLY OF APPLIED MATHEMATICS

VOLUME LXX, NUMBER 4

DECEMBER 2012, PAGES 773-786

S 0033-569X(2012)01272-9

Article electronically published on July 18, 2012

\title{
NON-EQUILIBRIUM RELATIVISTIC TWO-PHASE FLOW WITH HEAT EXCHANGE
}

\author{
BY \\ SEBASTIANO GIAMBÒ (Department of Mathematics, University of Messina, Viale F. Stagno \\ d'Alcontres, 31, 98166 Messina, Italy) \\ AND \\ GIUSEPPA MUSCIANISI (Department of Mathematics, University of Messina, Viale F. Stagno \\ d'Alcontres, 31, 98166 Messina, Italy)
}

Abstract. We consider two different systems of hyperbolic balance laws governing relativistic two-phase flows with general equations of state. The phases are assumed to be immiscible. In the first one the thermal equilibrium is attained through a relaxation procedure consistent with the second law of thermodynamics, while the second is a fully relaxed model in which the two phases reach an equal temperature instantaneously.

Finally, we deduce and compare the wave velocities for both the models: the hydrodynamical velocity of the relaxed system can never exceed that of the relaxation system.

1. Introduction. The development of models for compressible two-phase flows in both the classical and relativistic frameworks is really interesting in many scientific and engineering disciplines.

In the last few decades, several models have been developed both in classical 1, 2, 3, 10, 13, 23, 28, 29, 30, 31, 32, 33, 34, 35, 36, 40, 42, 43, and in relativistic [5, 6, 17, 8, 9, 11, 12, 14, 15, 16, 17, 18, 19, 20, 21, 22, 24, 25, 26, 37, 38, 39, 41, 44, formulation, but none of these models has been universally accepted as a complete formulation for modelling two-phase flows.

Both the classical and the relativistic mathematical modelling have certain inherent difficulties that originate from the existence of interfaces among the phases or the fluids. These interfaces separate not only pure media, but also mixtures of materials in which wave dynamics is important. Such situations appear frequently in astrophysics, the physics of explosives, nuclear physics and many other applications.

Received February 22, 2011.

2010 Mathematics Subject Classification. Primary 76T10, 76D33, 83A05.

The first author's work was supported by G.N.F.M. of I.N.d.A.M., by TirrenoAmbiente S.p.A. and by research grants of the University of Messina.

E-mail address: sgiambo@unime.it

E-mail address: gmuscianisi@unime.it

(C) 2012 Brown University

Reverts to public domain 28 years from publication 
The explained difficulties are concerned with the interaction between the fluids, which includes the transfer of mass, momentum and energy across the interfaces. The way in which such interfaces are treated is the key point of each model. To circumvent these difficulties, two classes of methods have been developed: the first class represents the models considering the interface as a sharp discontinuity; the second class corresponds to the models describing the interface as a diffused zone, such as the contact discontinuities in gas dynamics. The determination of thermodynamic flow variables in these zones is achieved on the basis of multiphase flow theory. The challenge is to derive physically and mathematically consistent thermodynamic laws for the mixture.

Following the ideas of Flåtten et al. [13, in the present paper we develop a new model for a relativistic two-phase diffusive flow with a single four-velocity and a single pressure, in which the two phases may coexist without being in thermal equilibrium. The mixture can be thought of as a continuum medium. By allowing the heat-transfer between the two phases, the mixture is driven towards the thermal equilibrium. This procedure is also referred to as a relaxation procedure, and such a system is also referred to as a relaxation system. As described in the next sections, the relaxation procedure adopted here is consistent with both the first and the second laws of thermodynamics.

Moreover, we consider the relaxed limit for the flow under investigation, in which the thermal equilibrium is supposed to be instantaneously reached. A very well-known relativistic flow model in the fully relaxed limit is the homogeneous equilibrium relativistic model, described and analysed for example in [15, 16.

In the model presented here, each fluid component still has its own particle number density, $r_{k}$, its specific internal energy, $\varepsilon_{k}$, and its energy density, $\rho_{k}, 4$, 27.

$$
\rho_{k}=r_{k}\left(1+\varepsilon_{k}\right), \quad k=1,2 .
$$

In what follows, the units are such that the velocity of light is unitary: $c=1$. Conversely, as mentioned above, we assume for the two phases of the fluid a single pressure, $p$, and a single four-velocity, $u^{\alpha}$, which is supposed to be future pointing:

$$
g_{\alpha \beta} u^{\alpha} u^{\beta}=1,
$$

where $g_{\alpha \beta}$ are the covariant components of the Lorentz metric tensor with signature + , ,,--- .

The paper is organized as follows. In Sect. 2 we describe the two-phase relativistic flow and we derive the flow equations. In Sect. 3 we introduce the relaxation procedure involving individual temperatures, one for each phase of the mixture, and we derive the necessary and sufficient restrictions on the relaxation terms imposed by the first and second laws of thermodynamics. Moreover, we deduce the expressions for the source terms appearing in the flow equations. In order to obtain the closure of the relaxation

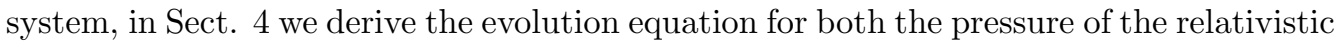
two-phase flow and for the volume fraction of a single phase of the mixture. In Sect. 5 we study the propagation of the weak discontinuity waves admitted by the relaxation system, obtaining the expression for the hydrodynamical mixture velocity. In Sect. 6 we analyse the fully relaxed system and we study the propagation of the weak discontinuity 
waves admitted by such a system. Finally, the wave velocities obtained both for the relaxation and the relaxed systems are compared.

2. Relativistic two-phase flow model. The relativistic two-phase flow model analysed in the present paper takes into account the balance laws for the particle number density of each phase:

$$
\nabla_{\alpha}\left(X_{k} r_{k} u^{\alpha}\right)=0, \quad k=1,2,
$$

and of the total energy-momentum conservation:

$$
\nabla_{\alpha} T^{\alpha \beta}=0
$$

where the stress energy tensor, $T^{\alpha \beta}$, is given by

$$
T^{\alpha \beta}=r f u^{\alpha} u^{\beta}-p g^{\alpha \beta} .
$$

Here, $r$ is the total particle number density, $X_{1}$ and $X_{2}$ are the volume fractions of the two phases of the mixture, such that $X_{1}+X_{2}=1, f$ is the total relativistic specific enthalpy

$$
f=1+h=1+\varepsilon+\frac{p}{r},
$$

where $h=\varepsilon+p / r$ and $\varepsilon$ represent, respectively, the "classical" specific enthalpy and the total energy density of the flow.

In order to determine suitable expressions for the bulk quantities $r, \varepsilon$ and $f$, we introduce the mass fraction $Y_{k}$ of the $k$-th phase, given by

$$
Y_{k}=\frac{X_{k} r_{k}}{r}, \quad k=1,2 .
$$

Taking into account the balance laws (2.1), the mass fractions of both the phases satisfy the relation $Y_{1}+Y_{2}=1$.

We define the total particle number density $r$ and the total specific internal energy $\varepsilon$ in terms of the mass and the volume fractions:

$$
\begin{aligned}
& r=X_{1} r_{1}+X_{2} r_{2}=X r_{1}+(1-X) r_{2}, \\
& \varepsilon=Y_{1} \varepsilon_{1}+Y_{2} \varepsilon_{2}=Y \varepsilon_{1}+(1-Y) \varepsilon_{2},
\end{aligned}
$$

where we set

$$
\begin{aligned}
& X_{1}=X, \quad X_{2}=1-X, \\
& Y_{1}=Y, \quad Y_{2}=1-Y .
\end{aligned}
$$

Thus, the bulk quantities $\rho=r(1+\varepsilon)$, i.e. the total energy density, $f$ and $r f$ can be easily expressed in terms of $X_{k}$ and $Y_{k}$ :

$$
\begin{aligned}
\rho & =X_{1} \rho_{1}+X_{2} \rho_{2}, \\
f & =Y_{1} f_{1}+Y_{2} f_{2}, \\
r f & =X_{1} r_{1} f_{1}+X_{2} r_{2} f_{2},
\end{aligned}
$$

where $f_{k}=1+\varepsilon_{k}+p / r_{k}$, for $k=1,2$.

The balance law for the total particle number density is given by

$$
\nabla_{\alpha}\left(r u^{\alpha}\right)=0
$$


where equations (2.1) and (2.6) are taken into account. Using (2.1), (2.5) and (2.13), we obtain the evolution equations for the mass fractions $Y_{k}$,

$$
u^{\alpha} \partial_{\alpha} Y_{k}=0, \quad k=1,2 .
$$

By virtue of the expression (2.12), we can rewrite the total energy-momentum tensor (2.3) as

$$
T^{\alpha \beta}=X_{1}\left(r_{1} f_{1} u^{\alpha} u^{\beta}-p g^{\alpha \beta}\right)+X_{2}\left(r_{2} f_{2} u^{\alpha} u^{\beta}-p g^{\alpha \beta}\right),
$$

and, thus, the evolution equation for the energy-momentum tensor of the $k$-th phase of the flow is

$$
\nabla_{\alpha}\left[X_{k}\left(r_{k} f_{k} u^{\alpha} u^{\beta}-p g^{\alpha \beta}\right)\right]=F_{k}^{\beta},
$$

where the $F_{k}^{\beta}$ represent the source term in the balance equation of each phase. According to the total energy-momentum conservation equation (2.2), the source terms $F_{k}^{\beta}$ are such that

$$
F_{1}^{\beta}=-F_{2}^{\beta}=F^{\beta} .
$$

The projection along $u^{\alpha}$, also referred to as the "energy evolution equation", and the spatial projection of the equation (2.16) for the fluid $k$ are, respectively,

$$
\begin{aligned}
& X_{k} r_{k} u^{\alpha}\left(\partial_{\alpha} \varepsilon_{k}+p \partial_{\alpha} \frac{1}{r_{k}}\right)-p u^{\alpha} \partial_{\alpha} X_{k}=u_{\alpha} F_{k}^{\alpha}, \\
& X_{k}\left(r_{k} f_{k} u^{\alpha} \nabla_{\alpha} u^{\beta}-\gamma^{\alpha \beta} \partial_{\alpha} p\right)-p \gamma^{\alpha \beta} \partial_{\alpha} X_{k}=\gamma_{\alpha}^{\beta} F_{k}^{\alpha},
\end{aligned}
$$

where $\gamma^{\alpha \beta}=g^{\alpha \beta}-u^{\alpha} u^{\beta}$ is the projection tensor onto the three-dimensional space orthogonal to $u^{\alpha}$ (i.e. the rest space of an observer moving with the four-velocity $u^{\alpha}$ ).

3. The relaxation procedure and the derivation of the source terms. The aim of this section is the handling of the source terms $F^{\beta}$, given by (2.17), appearing in the balance equations (2.16), and (2.18), applying relaxation heat-transfer terms between the phases of the mixture.

We assume that the entropy $S_{k}$ of each phase of the fluid is a function of both the internal energy $\varepsilon_{k}$ and the specific volume $1 / r_{k}$,

$$
S_{k}=S_{k}\left(\varepsilon_{k}, r_{k}\right), \quad k=1,2 .
$$

By thermodynamic argument, it follows that the derivatives of the entropy can be related to some observables

$$
\left(\frac{\partial S_{k}}{\partial \varepsilon_{k}}\right)_{r_{k}}=\frac{1}{T_{k}}, \quad\left(\frac{\partial S_{k}}{\partial r_{k}}\right)_{\varepsilon_{k}}=-\frac{p}{r_{k}^{2} T_{k}}, \quad k=1,2,
$$

where $T_{k}$ is the temperature of the phase $k$. From the above equations (3.2), we derive the following thermodynamic differential relations

$$
T_{k} d S_{k}=d \varepsilon_{k}+p d \frac{1}{r_{k}}, \quad k=1,2 .
$$

Thus, the following differential relations hold for the covariant derivative of $S_{k}$ :

$$
T_{k} u^{\alpha} \partial_{\alpha} S_{k}=u^{\alpha}\left(\partial_{\alpha} \varepsilon_{k}+p \partial_{\alpha} \frac{1}{r_{k}}\right), \quad k=1,2
$$


We consider a relaxation procedure in which 1) the heat should be transferred between the phases with a rate proportional to the difference of their temperatures, and 2) the variation of the entropy of each phase should be due only to the heat-transfer terms. Mathematically,

$$
\begin{aligned}
& X_{1} r_{1} T_{1} u^{\alpha} \partial_{\alpha} S_{1}=H_{12}\left(T_{2}-T_{1}\right), \\
& X_{2} r_{2} T_{2} u^{\alpha} \partial_{\alpha} S_{2}=H_{21}\left(T_{1}-T_{2}\right),
\end{aligned}
$$

where the relaxation coefficients, $H_{12}$ and $H_{21}$, are further assumed to be independent from the temperatures $T_{1}$ and $T_{2}$.

In order to develop a model consistent with the first and the second laws of thermodynamics, we compute that the relaxation coefficients have to satisfy the constraint

$$
H_{12}=H_{21}=H \geq 0 .
$$

In order to demonstrate the condition (3.7), we first observe that the total specific entropy of the mixture $S$ can be written in terms of the mass fractions of the two fluid phases,

$$
S=Y_{1} S_{1}+Y_{2} S_{2}
$$

and then, taking into account equations (2.14), (3.5) and (3.6), the evolution equation for the total entropy $S$ is given by

$$
r u^{\alpha} \partial_{\alpha} S=H_{12} \frac{T_{2}-T_{1}}{T_{1}}+H_{21} \frac{T_{1}-T_{2}}{T_{2}} .
$$

Equation (3.9) can be rewritten in a more convenient form,

$$
\nabla_{\alpha}\left(r S u^{\alpha}\right)=H_{12} \frac{\left(T_{2}-T_{1}\right)^{2}}{T_{1} T_{2}}+\left(H_{12}-H_{21}\right) \frac{T_{2}-T_{1}}{T_{2}}
$$

where the balance law for the total particle number (2.13) is used. Thus, (3.10) remains unconditionally non-negative if and only if the constraint (3.7) holds.

Introducing equations (3.4), (3.5) and (3.6) into the energy evolution equation (2.18), we obtain, under condition (3.7), that

$$
p u^{\alpha} \partial_{\alpha} X+u^{\alpha} F_{\alpha}=H\left(T_{2}-T_{1}\right),
$$

and thus, the source term $F_{\alpha}$ is equal to

$$
F_{\alpha}=-p \partial_{\alpha} X+H\left(T_{2}-T_{1}\right) u^{\alpha} .
$$

4. Evolution equations for total pressure and volume fraction. In this section we derive the evolution equation for the pressure of the mixture, and then the evolution equation for the volume fraction $X$.

Assuming that the mixture is isobaric, e.g.

$$
p=p_{k}\left(r_{k}, S_{k}\right)=p_{k}\left[r_{k}, \varepsilon_{k}\left(r_{k}, S_{k}\right)\right], \quad k=1,2,
$$

where $p_{k}$ is the pressure of each phase, we write the following differential formula:

$$
d p=d p_{k}=f_{k} \lambda_{k}^{2} d r_{k}+\Gamma_{k} r_{k} T_{k} d S_{k},
$$


where $\lambda_{k}$ represents the velocity of the single-phase hydrodynamical wave:

$$
\lambda_{k}^{2}=\left(\frac{\partial p}{\partial \rho_{k}}\right)_{S_{k}}=\frac{1}{f_{k}}\left(\frac{\partial p}{\partial r_{k}}\right)_{S_{k}}, \quad k=1,2,
$$

and $\Gamma_{k}$ is the Grüneisen coefficient of the $k$-th phase

$$
\Gamma_{k}=\frac{1}{r_{k}}\left(\frac{\partial p}{\partial \varepsilon_{k}}\right)_{r_{k}}, \quad k=1,2 .
$$

From (4.2), by using (3.5) and (3.6), we obtain the following equations:

$$
\begin{aligned}
& u^{\alpha} \partial_{\alpha} p=f_{1} \lambda_{1}^{2} u^{\alpha} \partial_{\alpha} r_{1}+\frac{1}{X_{1}} \Gamma_{1} H\left(T_{2}-T_{1}\right), \\
& u^{\alpha} \partial_{\alpha} p=f_{2} \lambda_{2}^{2} u^{\alpha} \partial_{\alpha} r_{2}+\frac{1}{X_{2}} \Gamma_{2} H\left(T_{1}-T_{2}\right),
\end{aligned}
$$

which using (2.1), can be rewritten as

$$
\begin{aligned}
\frac{X_{1}}{r_{1} f_{1} \lambda_{1}^{2}} u^{\alpha} \partial_{\alpha} p+\nabla_{\alpha}\left(X_{1} u^{\alpha}\right) & =\frac{\Gamma_{1}}{r_{1} f_{1} \lambda_{1}^{2}} H\left(T_{2}-T_{1}\right), \\
\frac{X_{2}}{r_{2} f_{2} \lambda_{2}^{2}} u^{\alpha} \partial_{\alpha} p+\nabla_{\alpha}\left(X_{2} u^{\alpha}\right) & =\frac{\Gamma_{2}}{r_{2} f_{2} \lambda_{2}^{2}} H\left(T_{1}-T_{2}\right) .
\end{aligned}
$$

From (4.7)-(4.8) we deduce the evolution equation for the pressure $p$,

$$
u^{\alpha} \partial_{\alpha} p+\frac{1}{\omega} \nabla_{\alpha} u^{\alpha}=\frac{\xi}{\omega} H\left(T_{2}-T_{1}\right),
$$

where

$$
\begin{gathered}
\omega=\frac{X_{1}}{r_{1} f_{1} \lambda_{1}^{2}}+\frac{X_{2}}{r_{2} f_{2} \lambda_{2}^{2}}, \\
\xi=\frac{\Gamma_{1}}{r_{1} f_{1} \lambda_{1}^{2}}-\frac{\Gamma_{2}}{r_{2} f_{2} \lambda_{2}^{2}} .
\end{gathered}
$$

The evolution equation for the volume fraction $X_{1}=X$ is easily obtained by introducing equation (4.9) into (4.7),

$$
u^{\alpha} \partial_{\alpha} X+X(1-X) \zeta \nabla_{\alpha} u^{\alpha}=\eta H\left(T_{2}-T_{1}\right),
$$

where

$$
\begin{gathered}
\zeta=\frac{r_{1} f_{1} \lambda_{1}^{2}-r_{2} f_{2} \lambda_{2}^{2}}{X_{2} r_{1} f_{1} \lambda_{1}^{2}+X_{1} r_{2} f_{2} \lambda_{2}^{2}}, \\
\eta=\frac{X_{2} \Gamma_{1}+X_{1} \Gamma_{2}}{X_{2} r_{1} f_{1} \lambda_{1}^{2}+X_{1} r_{2} f_{2} \lambda_{2}^{2}} .
\end{gathered}
$$

Therefore, the complete relaxation system describing the evolution of the two-phase relativistic flow under investigation is given by the following system of eight differential 
equations in the eight independent field variables $u^{\alpha}, r_{1}, r_{2}, S_{1}, S_{2}, X$ :

$$
\left\{\begin{array}{l}
r f u^{\alpha} \nabla_{\alpha} u^{\beta}-\gamma^{\alpha \beta} \partial_{\alpha} p=0, \\
\nabla_{\alpha}\left[X r_{1} u^{\alpha}\right]=0 \\
\nabla_{\alpha}\left[(1-X) r_{2} u^{\alpha}\right]=0 \\
u^{\alpha} \partial_{\alpha} S_{1}=\frac{1}{X r_{1} T_{1}} H\left(T_{2}-T_{1}\right), \\
u^{\alpha} \partial_{\alpha} S_{2}=\frac{1}{(1-X) r_{2} T_{2}} H\left(T_{1}-T_{2}\right), \\
u^{\alpha} \partial_{\alpha} X+X(1-X) \zeta \nabla_{\alpha} u^{\alpha}=\eta H\left(T_{2}-T_{1}\right),
\end{array}\right.
$$

where $\omega, \xi, \zeta$ and $\eta$ are given by (4.10), 4.11), (4.13) and (4.14). In addition to such a system (4.15), the evolution equation (4.9) for the pressure $p$ of the mixture, $p=$ $p_{1}\left(r_{1}, S_{1}\right)=p_{2}\left(r_{2}, S_{2}\right)$, holds.

5. Weak discontinuity wave propagation for the relaxation system. In a domain $\mathcal{D}$ of the space-time $V_{4}$, we consider a regular hypersurface, $\Sigma$, not generated by the flow lines, with local equation $\varphi\left(x^{\alpha}\right)=0$. As will be clear below, the hypersurface $\Sigma$ is a space-like one, i.e. $L^{\alpha} L_{\alpha}<0$, where $L_{\alpha}=\partial_{\alpha} \varphi$. In what follows, we denote by $N_{\alpha}$ the normalized vector

$$
N_{\alpha}=\frac{L_{\alpha}}{\sqrt{-L^{\beta} L_{\beta}}}, \quad N_{\alpha} N^{\alpha}=-1
$$

We consider a particular class of solutions of system (4.15), namely, weak discontinuity waves $\Sigma$, on which the independent field variables $u^{\alpha}, r_{1}, r_{2}, S_{1}, S_{2}$ and $X$ are continuous, but jump discontinuities may occur in their normal derivatives. In this case, if $Q$ denotes any of the fields, then there exists [4, 27] the distribution $\delta Q$, with support $\Sigma$, such that

$$
\bar{\delta}\left[\nabla_{\alpha} Q\right]=N_{\alpha} \delta Q
$$

where $\bar{\delta}$ is the Dirac measure defined by $\varphi$ with $\Sigma$ as support and square brackets denote the discontinuity, $\delta$ being an operator of infinitesimal discontinuity; $\delta$ behaves like a derivative insofar as algebraic manipulations are concerned. 
From system (4.15), we obtain the following linear homogeneous system in the distributions $\delta u^{\alpha}, \delta r_{1}, \delta r_{2}, \delta S_{1}, \delta S_{2}$ and $\delta X$ :

$$
\left\{\begin{array}{l}
r f L \delta u^{\alpha}-\gamma^{\alpha \beta} N_{\beta} \delta p=0 \\
X r_{1} N_{\alpha} \delta u^{\alpha}+X L \delta r_{1}+r_{1} L \delta X=0, \\
(1-X) r_{2} N_{\alpha} \delta u^{\alpha}+(1-X) L \delta r_{2}-r_{2} L \delta X=0 \\
L \delta S_{1}=0 \\
L \delta S_{2}=0 \\
L \delta X+X(1-X) \zeta N_{\alpha} \delta u^{\alpha}=0
\end{array}\right.
$$

where $L=u^{\alpha} N_{\alpha}$, and from equation (4.9) we obtain the following equation for the distribution $\delta p$ :

$$
\omega L \delta p+N_{\alpha} \delta u^{\alpha}=0 .
$$

Due to the unitary character of $u^{\alpha}$, we also have that

$$
u_{\alpha} \delta u^{\alpha}=0 \text {. }
$$

In what follows, we investigate the normal speeds of propagation of the various waves with respect to an observer moving with the flow velocity $u^{\alpha}$. The normal speed $\lambda_{\Sigma}$ of propagation of the wave front $\Sigma$, described by a time-like word line having tangent vector field $u^{\alpha}$, that is, the velocity in the time direction $u^{\alpha}$, is given by [4, 27]

$$
\lambda_{\Sigma}^{2}=\frac{L^{2}}{1+L^{2}} .
$$

The local causality condition, i.e. the requirement that the characteristic hypersurface $\Sigma$ has to be time-like or null (or equivalently that the normal $N_{\alpha}$ be space-like or null, $g^{\alpha \beta} N_{\alpha} N_{\beta} \leq 0$ ) is equivalent to the condition $0 \leq \lambda_{\Sigma}^{2} \leq 1$.

From the above system (5.3), we first obtain the solution in the case $L=0$, which represents a wave moving with the mixture. The corresponding discontinuities are characterized by the conditions

$$
\delta p=0, \quad N_{\alpha} \delta u^{\alpha}=0 ;
$$

this implies that system (5.3) admits six independent eigenvectors corresponding to $L=0$ in the space of the field variables.

From now on, we suppose $L \neq 0$. Thus, by $(\underline{5.3})_{4,5}$, we obtain that $\delta S_{1}=\delta S_{2}=0$. Equation (5.3) ${ }_{1}$ multiplied by $N_{\alpha}$ gives us

$$
\operatorname{rfL} N_{\alpha} \delta u^{\alpha}+l^{2} \delta p=0,
$$

where $l^{2}=1+L^{2}$. As a consequence, equations (5.3) 2 and (5.8) represent a linear homogeneous system, in the two scalar distributions $N_{\alpha} \delta u^{\alpha}$ and $\delta p$, which may have non-trivial solutions only if the determinant of the coefficient vanishes, i.e.

$$
\mathcal{H} \equiv \omega r f L^{2}-l^{2}=0,
$$


which corresponds to the hydrodynamical waves propagating in such a two-fluid relaxation system. Their speed of propagation is given by

$$
\lambda_{\Sigma}^{2}=\frac{1}{\omega r f}=\frac{1}{r f\left\{\frac{X_{1}}{r_{1} f_{1} \lambda_{1}^{2}}+\frac{X_{2}}{r_{2} f_{2} \lambda_{2}^{2}}\right\}},
$$

and the local causality condition, which ensures their spatial orientation, is given by

$$
0<\frac{1}{\omega r f} \leq 1
$$

Moreover, from (5.10), the following relation holds:

$$
\frac{1}{r f \lambda_{\Sigma}^{2}}=\frac{X_{1}}{r_{1} f_{1} \lambda_{1}^{2}}+\frac{X_{2}}{r_{2} f_{2} \lambda_{2}^{2}}
$$

The associated discontinuities can be written in terms of $\psi=n_{\alpha} \delta u^{\alpha}$ :

$$
\left\{\begin{array}{l}
\delta u^{\alpha}=-\psi n^{\alpha} \\
\delta r_{1}=-r_{1} \frac{l}{L}[1-\zeta(1-X)] \psi, \quad \delta r_{2}=-r_{2} \frac{l}{L}[1+X \zeta] \psi \\
\delta S_{1}=0, \quad \delta S_{2}=0 \\
\delta X=-\frac{-(1-X) X \zeta}{L} l \psi, \quad \delta p=-\frac{l}{\omega L} \psi
\end{array}\right.
$$

where $n^{\alpha}$ is the unitary space-like four-vector defined by

$$
n_{\alpha}=\frac{1}{l}\left(N_{\alpha}-L u_{\alpha}\right)
$$

If the above condition (5.11), characterizing the space-like orientations of the surfaces, is verified, the differential equations (4.15), governing the motion of the two-fluid relaxation system, represent a (not strictly) hyperbolic system. In fact, all velocities (eigenvalues) are real, and there exists a complete set of eigenvectors in the space of field variables, i.e. eight independent eigenvectors ( $\operatorname{six}$ from $L=0$ and two from $\mathcal{H}_{1}=0$ ), for the eight independent field variables $u^{\alpha}, r_{1}, r_{2}, S_{1}, S_{2}$ and $X$.

6. Relaxed system. We now consider the system obtained by letting the relaxation coefficient $H$ tend to infinity; i.e., we achieve instantaneous thermal equilibrium among the two phases of the mixture. Assuming the same temperature for the two phases, $T_{1}=T_{2}$, the entropy of the mixture, given by equation (3.8), satisfies the characteristic equation

$$
u^{\alpha} \partial_{\alpha} S=0,
$$

where relations (2.13) and (3.10) have been taken into account. 
The relaxed system may be expressed in a convenient form as

$$
\left\{\begin{array}{l}
r f u^{\alpha} \nabla_{\alpha} u^{\beta}-\gamma^{\alpha \beta} \partial_{\alpha} p=0, \\
\nabla_{\alpha}\left(r u^{\alpha}\right)=0 \\
u^{\alpha} \partial_{\alpha} S=0 \\
u^{\alpha} \partial_{\alpha} Y=0 .
\end{array}\right.
$$

Moreover, by virtue of equations (2.5), (2.6), (2.7), (3.3) and (3.8), and setting a common value for the temperatures of the two phases, $T=T_{1}=T_{2}$, we can write the following generalized Gibbs equation:

$$
d \varepsilon=T d S-p d \frac{1}{r}-\left\{\left(\varepsilon_{2}+\frac{p}{r_{2}}-T S_{2}\right)-\left(\varepsilon_{1}+\frac{p}{r_{1}}-T S_{1}\right)\right\} d Y,
$$

and, thus, we can suppose that the equation of state for the pressure of the mixture is given by $p=p(r, S, Y)$. Thus, (6.2) is a system of six differential equations in the six independent field variables $u^{\alpha}, r, S$ and $Y$.

In order to study a weak discontinuity waves propagation compatible with the relaxed system (6.2), we apply the equation (5.2) to the system (6.2). Thus we obtain the following linear homogeneous system in the distributions $\delta u^{\alpha}, \delta r, \delta S$ and $\delta Y$ :

$$
\left\{\begin{array}{l}
r f L \delta u^{\beta}-\gamma^{\alpha \beta} N_{\alpha}\left\{\left(\frac{\partial p}{\partial r}\right)_{S, Y} \delta r+\left(\frac{\partial p}{\partial S}\right)_{r, Y} \delta S+\left(\frac{\partial p}{\partial Y}\right)_{r, S} \delta Y\right\}=0, \\
L \delta r+r N_{\alpha} \delta u^{\alpha}=0, \\
L \delta S=0, \\
L \delta Y=0 .
\end{array}\right.
$$

From the above equations (6.4), if $L=0$, we have $N_{\alpha} \delta u^{\alpha}=\delta p=0$ and thus system (6.4) admits four independent eigenvectors in the space of the field variables.

In the case $L \neq 0$, equations (6.4 $)_{3,4}$ give $\delta S=\delta Y=0$. By composing the equation (6.4) 1 with $N_{\beta}$ we obtain

$$
r f L N_{\alpha} \delta u^{\alpha}+l^{2}\left(\frac{\partial p}{\partial r}\right)_{S, Y} \delta r=0
$$

which, together with the equation $(6.4)_{2}$, represents a linear homogeneous system in the two scalar distributions $N_{\alpha} \delta u^{\alpha}$ and $\delta r$. This system has non-trivial solutions iff

$$
\tilde{\mathcal{H}}=f L^{2}-l^{2}\left(\frac{\partial p}{\partial r}\right)_{S, Y}=0
$$

which corresponds to the hydrodynamical waves. Their speeds of propagation are given by

$$
\tilde{\lambda}_{\Sigma}^{2}=\frac{1}{f}\left(\frac{\partial p}{\partial r}\right)_{S, Y}
$$


Since the pressure of such a system is given by

$$
p(r, S, Y)=p[\rho(r, S, Y), S, Y],
$$

and taking into account that

$$
\left(\frac{\partial \rho}{\partial r}\right)_{S, Y}=f
$$

we obtain

$$
\left(\frac{\partial p}{\partial r}\right)_{S, Y}=\left(\frac{\partial p}{\partial \rho}\right)_{S, Y}\left(\frac{\partial \rho}{\partial r}\right)_{S, Y}=f\left(\frac{\partial p}{\partial \rho}\right)_{S, Y} .
$$

Thus the speeds (6.7) can be rewritten as

$$
\tilde{\lambda}_{\Sigma}^{2}=\left(\frac{\partial p}{\partial \rho}\right)_{S, Y} .
$$

As we shall demonstrate in the following, the mixture hydrodynamical velocity of the relaxed system is always less than the corresponding velocity of the relaxation system, e.g.

$$
0<\tilde{\lambda}_{\Sigma}^{2} \leq \lambda_{\Sigma}^{2} \leq 1
$$

and by the local causality condition (5.11), we can conclude that the wave surfaces for the relaxed system are time-like.

The associated discontinuities to the distribution in the system (6.4), written in terms of $\tilde{\psi}=n_{\alpha} \delta u^{\alpha}$, are

$$
\left\{\begin{array}{l}
\delta u^{\alpha}=-\tilde{\psi} n^{\alpha} \\
\delta r=-r \frac{l}{L} \tilde{\psi} \\
\delta S=0 \\
\delta Y=0
\end{array}\right.
$$

where $n^{\alpha}$ is the unitary space-like four-vector given by (5.14).

Since the condition characterizing the space-like orientations of the surface is verified, the governing equations represent a (not strictly) hyperbolic system. All velocities (eigenvalues) are real, and there exists a complete set of eigenvectors in the space of field variables, i.e. six independent eigenvectors (four from $L=0$ and two from $\tilde{\mathcal{H}}=0$ ), for the six independent field variables $u^{\alpha}, r, S, Y$.

In order to prove inequality (6.12), we rewrite any thermodynamic relation in terms of the parameters $\zeta_{k}$ defined as

$$
\zeta_{k}=\left(\frac{\partial T}{\partial p}\right)_{S_{k}}=-\frac{1}{r_{k}^{2}}\left(\frac{\partial r_{k}}{\partial S_{k}}\right)_{p}, \quad k=1,2 .
$$

Thus we obtain that

$$
\left(\frac{\partial S_{k}}{\partial p}\right)_{T}=\frac{1}{r_{k}^{2}}\left(\frac{\partial r_{k}}{\partial T}\right)_{p}=-\frac{\zeta_{k} \hat{C}_{k}}{T}, \quad k=1,2,
$$


where the specific heat capacity $\hat{C}_{k}$ of each phase of the mixture is given by

$$
\hat{C}_{k}=T\left(\frac{\partial S_{k}}{\partial T}\right)_{p}, \quad k=1,2 .
$$

Following the idea of Flåtten, Morin and Munkejord [13, we consider the differential

$$
D=\frac{1}{r_{1}} d\left(X_{1} r_{1}\right)+\frac{1}{r_{2}} d\left(X_{2} r_{2}\right)=\frac{X_{1}}{r_{1}} d r_{1}+\frac{X_{2}}{r_{2}} d r_{2},
$$

which in a more explicit form is

$$
D=\frac{X_{1}}{r_{1}}\left[\left(\frac{\partial r_{1}}{\partial p}\right)_{S_{1}} d p+\left(\frac{\partial r_{1}}{\partial S_{1}}\right)_{p} d S_{1}\right]+\frac{X_{2}}{r_{2}}\left[\left(\frac{\partial r_{2}}{\partial p}\right)_{S_{2}} d p+\left(\frac{\partial r_{2}}{\partial S_{2}}\right)_{p} d S_{2}\right],
$$

and, by virtue of (4.3), (5.12) and (6.14), it can be written as

$$
D=\frac{1}{r f \lambda_{\Sigma}^{2}} d p-\left(X_{1} r_{1} \zeta_{1} d S_{1}+X_{2} r_{2} \zeta_{2} d S_{2}\right) .
$$

Taking into account that the entropy of each phase of the mixture is a function $S_{k}=$ $S_{k}(p, T)$, and using the thermodynamic relations (6.15) and (6.16), the differential (6.19) can also be written as

$$
D=\left[\frac{1}{r f \lambda_{\Sigma}^{2}}+\frac{\zeta_{1}^{2} C_{1}+\zeta_{2}^{2} C_{2}}{T}\right] d p-\frac{\zeta_{1} C_{1}+\zeta_{2} C_{2}}{T} d T,
$$

where the extensive heat capacity $C_{k}$ of each phase of the mixture is given by

$$
C_{k}=X_{k} r_{k} \hat{C}_{k}=r Y_{k} \hat{C}_{k}, \quad k=1,2 .
$$

Furthermore, using (6.15) and (6.16), the differential of the total entropy $S$ of the mixture, defined in (3.8), is given by

$$
\begin{aligned}
d S & =Y_{1} d S_{1}+Y_{2} d S_{2}+O(d Y) \\
& =\frac{Y_{1} \hat{C}_{1}+Y_{2} \hat{C}_{2}}{T} d T-\frac{Y_{1} \zeta_{1} \hat{C}_{1}+Y_{2} \zeta_{2} \hat{C}_{2}}{T} d p+O(d Y) .
\end{aligned}
$$

Introducing the relation (6.22) into the expression of the differential (6.20) we obtain

$$
D=\left[\frac{1}{r f \lambda_{\Sigma}^{2}}+\frac{C_{1} C_{2}\left(\zeta_{1}-\zeta_{2}\right)^{2}}{T\left(C_{1}+C_{2}\right)}\right] d p-r \frac{\zeta_{1} C_{1}+\zeta_{2} C_{2}}{C_{1}+C_{2}} d S+O(d Y)
$$

Moreover, by virtue of relation (2.5) between the mass and the volume fractions, the differential $D$, defined in (6.17), can also be written as

$$
\begin{aligned}
D & =\frac{1}{r_{1}} d\left(X_{1} r_{1}\right)+\frac{1}{r_{2}}\left(X_{2} r_{2}\right)=\frac{1}{r_{1}} d\left(Y_{1} r_{1}\right)+\frac{1}{r_{2}} d\left(Y_{2} r\right) \\
& =\left(\frac{Y_{1}}{r_{1}}+\frac{Y_{2}}{r_{2}}\right) d r+O(d Y) \\
& =\frac{1}{r}\left\{\left(\frac{\partial r}{\partial p}\right)_{S, Y} d p+\left(\frac{\partial r}{\partial S}\right)_{p, Y} d S\right\}+O(d Y) \\
& =\frac{1}{r f \tilde{\lambda}_{\Sigma}^{2}} d p+\frac{1}{r}\left(\frac{\partial r}{\partial S}\right)_{p, Y} d S+O(d Y) .
\end{aligned}
$$


By comparing relations (6.23) and (6.24), the mixture hydrodynamical velocity of the relaxed system, $\tilde{\lambda}_{\Sigma}^{2}$, can be written in terms of the mixture hydrodynamical velocity of the relaxation system, $\lambda_{\Sigma}^{2}$, as

$$
\frac{1}{\tilde{\lambda}_{\Sigma}^{2}}-\frac{1}{\lambda_{\Sigma}^{2}}=\frac{r f}{T\left(C_{1}+C_{2}\right)} C_{1} C_{2}\left(\zeta_{1}-\zeta_{2}\right)^{2} .
$$

It is easily observed that the previous difference is non-negative only if the extensive heat capacities of the two components of the mixture are positive:

$$
C_{1}>0, \quad C_{2}>0,
$$

and the equality holds iff $\zeta_{1}$ and $\zeta_{2}$ are equal.

Thus, under the condition (6.26), the following inequality holds:

$$
0<\tilde{\lambda}_{\Sigma}^{2} \leq \lambda_{\Sigma}^{2} \leq 1
$$

i.e. the mixture hydrodynamical velocity of the relaxed system, $\tilde{\lambda}_{\Sigma}^{2}$, is space-like, since the mixture hydrodynamical velocity of the relaxation system, $\lambda_{\Sigma}^{2}$, satisfies the condition (5.11), which ensures its spatial orientation.

In conclusion, we can say that the absolute value of the velocities of the hydrodynamical waves of the relaxed system can never exceed those of the relaxation system.

\section{REFERENCES}

[1] R. Abgrall, How to prevent pressure oscillations in multicomponent flow calculations: a quasiconservative approach, J. Comput. Phys. 125 (1996), 150-160. MR.1381808(96k:76082)

[2] G. Allaire, S. Clerc, and S. Kokh, A five-equation model for the simulation of interfaces between compressible fluids, J. Comput. Phys. 181 (2002), 577-616. MR.1927402 (2003g:76085)

[3] N. Andrianov, R. Saurel, and G. Warnecke, A simple method for compressible multiphase mixtures and interfaces, Int. J. Numer. Meth. Fluids 41 (2003), 109-131. MR1950175 (2003m:76115)

[4] A. M. Anile, Relativistic fluids and magneto-fluids, Cambridge University Press, Cambridge, 1989.

[5] S. S. Bayin, Anisotropic fluid spheres in general relativity, Phys. Rev. D 26 (1982), 1262-1274. MR675034 (84j:83042)

[6] M. Cissoko, Wavefronts in a relativistic cosmic two-component fluid, Gen. Rel. Grav. 30 (1998), 521-534. MR.1618979

[7] __ Wave fronts in a mixture of two relativistic perfect fluids flowing with two distinct fourvelocities, Phys. Rev. D 63 (2001), 083516. MR.1831145 (2002b:83032)

[8] A. A. Coley and B. O. J. Tupper, Imperfect fluid cosmologies with thermodynamics - some exact solutions, Astrophys. J. 280 (1984), 26-33. MR743301 (85c:83104)

[9] , Two-fluid cosmological models, J. Math. Phys. 27 (1986), 406-416. MR816462 (87g:83150)

[10] V. Deledicque and M. V. Papalexandris, A conservative approximation to compressible two-phase flow models in the stiff mechanical relaxation limit, J. Comput. Phys. 227 (2008), 9241-9270. MR:2463206 (2009j:76177)

[11] K. Dunn, Two-fluid cosmological models in Gödel-type spacetimes, Gen. Rel. Grav. 21 (1998), 137-147. MR990147 (90e:83019)

[12] J. J. Ferrando, J. A. Morales, and M. Portilla, Two-perfect fluid interpretation of an energy tensor, Gen. Rel. Grav. 22 (1990), 1021-1032. MR.1067616 (91g:83039)

[13] T. Flătten, A. Morin, and S. T. Munkejord, Wave propagation in multicomponent flow models, SIAM J. Appl. Math. 70 (2010), no. 8, 2861-2882. MR.2735107

[14] V. E. Fortov and I. V. Lomonosov, Equations of state of matter at high energy densities, The Open Plasma Physics Journal 3 (2010), 122-130.

[15] S. Giambò and S. Giambò, A seven equation model for relativistic two fluid flows-I, ROMAI J. 5 (2009), 59-70. MR2721255

[16] , A seven equation model for relativistic two fluid flows-II, ROMAI J. 6 (2010), 95-105. $\operatorname{MR} 2739286$ 
[17] _ A model for relativistic fluid mixture with different pressure laws, EPL 90 (2010), 54004.

[18] E. N. Glass and J. P. Krisch, Two-fluid atmosphere for relativistic stars, Class. Quantum Grav. 16 (1999), 1175-1184. MR 1696147(2000c:83057)

[19] Yu. B. Ivanov, V. N. Russkikh, and V. D. Toneev, Relativistic heavy-ion collisions within three-fluid hydrodynamics: hadronic scenario, Phys. Rev. C 73 (2006), 044904.

[20] A. Krasiński, Inhomogeneous cosmological models, Cambridge University Press, Cambridge, 1997.

[21] M. Krause and P. Alexander, Simulations of multi-phase turbulence in jet cocoons, Mon. Not. R. Astron. Soc. 376 (2007), 465-478.

[22] J. P. Krisch and L. L. Smalley, Two fluid acoustic modes and inhomogeneous cosmologies, Class. Quantum Grav. 10 (1993), 2615-2623. MR.1252288 (94g:83077)

[23] F. Lagoutière, Modélisation mathématique et résolution numérique de problèmes de fluides compressible plusieurs constituants, Ph.D. thesis, Université de Paris VI, 2000.

[24] P. S. Letelier, Anisotropic fluids with two-perfect-fluid components, Phys. Rev. D 22 (1980), 807813. MR.583095 (81g:83039)

[25] _ Solitary waves of matter in general relativity, Phys. Rev. D 26 (1980), 2623-2631. MR686726 (84c:83015)

[26] P. S. Letelier and P. S. Alencar, Anisotropic fluids with multifluid components, Phys. Rev. D 34 (1986), 343-351. MR848075 (87g:83083)

[27] A. Lichnerowicz, Relativistic fluid dynamics, Cremonese, Roma, 1971. MR0297295 (45:6352)

[28] H. Lund and T. Flåtten, Equilibrium conditions and sound velocities in two-phase flows, SIAM Annual Meeting 2010 (Pittsburg, Pennsylvania, USA), 2010.

[29] S. Müller, P. Helluy, and J. Ballmann, Numerical simulation of cavitation bubbles by compressible two-phase fluids, Int. J. Fluid Mech. (2009).

[30] A. Murrone and H. Guillard, A five-equation reduced model for compressible two-phase flow problems, J. Comput. Phys. 202 (2005), 664-698. MR2145395 (2006e:76133)

[31] F. Petitpas, J. Massoni, R. Saurel, E. Lapebie, and L. Munier, Diffuse interface models for high speed cavitating underwater systems, Int. J. Multiphase Flows 35 (2009), 747-759.

[32] F. Petitpas, R. Saurel, E. Franquet, and A. Chinnayya, Modelling detonation waves in condensed materials: multiphase CJ conditions and multidimensional computations, Shock waves 19 (2009), 377-401.

[33] E. Romenski and E. F. Toro, Compressible two-phase flows: Two-pressure models and numerical methods, Comp. Fluid Dyn. J. 13 (2004), 403-416.

[34] R. Saurel, O. Le Metayer, J. Massoni, and S. Gavrilyuk, Shock jump relations for multiphase mixtures with stiff mechanical properties, Shock waves 16 (2007), 209-232.

[35] R. Saurel, F. Petitpas, and R. Abgrall, Modelling phase transition in metastable liquids: application to cavitating and flashing flows, J. Fluid Mech. 607 (2008), 313-350. MR2436919 (2009i:80011)

[36] R. Saurel, F. Petitpas, and R. A. Berry, Simple and efficient relaxation methods for interfaces separating compressible fluids, cavitating flows and shocks in multiphase mixtures, J. Comput. Phys. 228 (2009), 1678-1712. MR2494233 (2010b:76090)

[37] R. F. Sistero, Relativistic cosmological models with pressure, Astrophys. Space Sci. 12 (1971), 484.

[38] V. D. Toneev, Yu. B. Ivanov, E. G. Nikonov, W. Nörenberg, and V. N. Russkikh, Three-fluid simulations of relativistic heavy-ion collisions, Physics of Particles and Nuclei Letters 2(2) (2005), $43-49$.

[39] A. I. Voropinov and M. A. Podurets, Relativistic gravitational collapse of a cool white dwarf with allowance for the neutronization kinetics, Astronomy Letters 27 (2001), no. 6, 366-375.

[40] J. Wackers and B. Koren, A fully conservative model for compressible two-fluid flow, Int. J. Numer. Meth. Fluids 47 (2005), 1337-1343. MR2128761 (2005m:65183)

[41] R. D. Zárate and H. Quevedo, Thermodynamic scheme of inhomogeneous perfect fluid mixtures, Class. Quantum Grav. 21 (2004), 197-205. MR2026686 (2004m:83043)

[42] D. Zeidan, E. Romenski, A. Slaouti, and E. F. Toro, Numerical study of wave propagation in compressible two-phase flow, Int. J. Numer. Meth. Fluids 54 (2007), 393-417. MR2314750 (2008a:76128)

[43] A. Zein, M. Hantke, and G. Warnecke, Modeling phase transition for compressible two-phase flows applied to metastable liquids, J. Comput. Phys. 229 (2010), 2964-2998. MR2595804 (2011c:80007)

[44] W. Zimdahl, D. Pavon, and R. Maartens, Reheating and causal thermodynamics, Phys. Rev. D 55 (1997), 4861-4688. 